\title{
Discontinuity Adaptive MRF Model for Synthetic Aperture Radar Image Analysis
}

\author{
P.C. Smits ${ }^{1}$, S.G. Dellepiane ${ }^{1}$, and G. Vernazza ${ }^{2}$ \\ ${ }^{1}$ University of Genoa \\ Dept. Biophysical and Electronic Eng \\ Via all'Opera Pia 11A, Genova Italy \\ \{smits,silvana\}@dibe.unige.it \\ ${ }^{2}$ University of Cagliari \\ Dept. Electrical and Electronic Eng \\ Piazza d'Armi, Cagliari, Italy \\ vernazza@diee.unica.it
}

\begin{abstract}
In this paper, an approach is presented for the reconstruction and analysis of synthetic aperture radar (SAR) images that preserves better fine structures and borders in the image than classical methods. The method uses the discontinuity adaptive MRF model proposed by $\mathrm{Li}$ [1] in combination which an observation model that exploits a gamma distribution. This resulted in a new algorithm that is suited to the analysis of SAR images.
\end{abstract}

\section{Introduction and problem definition}

Image restoration, segmentation and classification of images can be formulated as ill-posed problems. Although the quality of many modern imaging sensors is such that these problems are not too ill-posed, this situation changes in cases where one wants to extract types of information for which the sensor has not been build in the first place. In order to tackle an ill-posed problem in such cases, a-priori constraints or other sources of information are important for the regularization of the problem. In this article, we focus on the problem of SAR intensity image restoration for land-cover mapping applications. This data may contain regions that have geometrically difficult shapes, like fine structures and critical borders between classes, that are obscured by speckle noise. Existing literature does address the incorporation of adequate statistical models in segmentation algorithms for this type of data, but fails to pay sufficient attention to the aspect of preserving small structures.

Markov Random Field (MRF) approaches have shown to be useful because of the ability to define the spatial interaction between the pixels in the image. In the literature, the interactions between pixels are fixed for the entire image, and may lead to undesired smoothing. This paper builds on two lines of research reported in the literature. One concerns aspects related to the statistics of SAR intensity data, and the other relates to recent developments in the use of a more precise MRF models. The novelty of this paper is the combination of a discontinuity adaptive (DA-) MRF model that accounts for small structures and discontinuities as proposed in $[1,2]$ and the Gamma distribution as the image model, which appeared to be useful for modeling SAR data [3].

This article is organized as follows. Section 2 gives an overview of the proposed method. Section 3 elucidates the observation model as presented in [3]. Section 4 outlines Li's DA-MRF model. How the observation and the 
regularization model are merged is explained in section 5. Section 6 shows some experimental results, followed by discussion and conclusions (section 7 ).

\section{Method overview}

In the approach proposed in this paper, an MRF model is utilized in which regularization constraints like smoothness are encoded into an energy following a probabilistic route. Basically, the model consists of the sum of two energy terms. One term is the observation model, which defines the relation between the observed intensity data and the image labels (see Fig. 1). The energy returned by this term is a measure for the closeness of the observation to an image class model. The second term is a regularizer, and penalizes the irregularities according to the a-priori smoothness constraint encoded in it (see Fig. 2).

Both energy terms have been object of study: the observation model since it has to cope with images degraded by speckle noise with its typical gamma distribution [3], and the regularizer because in many image analysis applications the assumption of the uniform smoothness everywhere can lead to undesirable, over smoothed, solutions ([2],[4]).

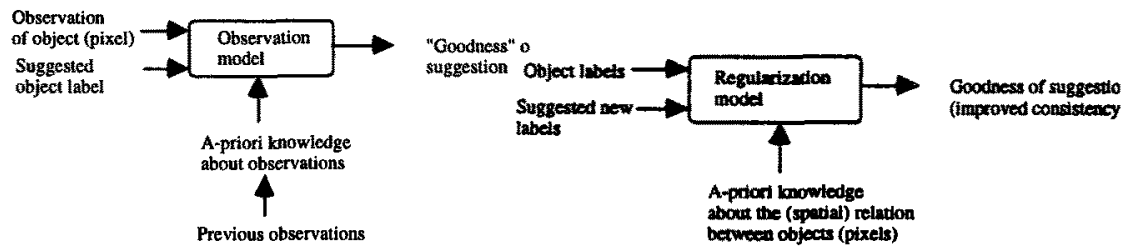

Fig. 1. Data flow diagram of the observation regularizer.

Fig. 2. Data flow diagram of the model.

\section{Observation model}

In this section, we are concerned with the observation model, i.e., the relation between the intensity data $I$ and our image labels $L$. In the literature agreement exists on the gamma distribution being one of the most suitable one for SAR data. The distribution for multilook intensities are modeled as [3]:

$$
p\left(I_{s} / L_{s}\right)=\frac{N^{N} I_{s}^{N-1}}{\langle I\rangle_{l}^{N} \Gamma(N)} \exp \left(-\frac{N I_{s}}{\langle I\rangle_{l}}\right)
$$

where $s$ is the index for the location, $I$ is the intensity of the SAR data, $L$ the label, $N$ the number of independent one-look samples used to form each multilook intensity sample $I_{s}$ and $\Gamma(N)=(N-1)$ !. The energy function $U_{1}$ is given by

$$
U_{1}\left(I_{s} / L_{s}\right)=\frac{N I_{s}}{\langle I\rangle_{L_{s}}}-(N-1) \log \left(I_{s}\right)+N \log \left(\langle I\rangle_{L_{s}}\right) \text {. }
$$


Equation (2) is the class-conditional energy function or image model that will be used in section 5. Basically it says that, given a class $L_{s}$ it returns a low value (good) if the intensity of a pixel under test $s$ is close to the mean intensity of the suggested class $L_{s}$, and a high value (not so good) if otherwise.

\section{Discontinuity adaptive MRF model}

The MAP labeling with a prior potential is equivalent to the regularization of an ill-posed problem. An important assumption of the classical implementation of a regularization constraint is the smoothness. It is incorporated into the energy function whereby the cost of the solution is defined. A regularized solution corresponds to the maximum a-posteriori estimate of an MRF.

In this article, the smoothness constraint is not blindly applied to an entire image, but the introduction of line processes or weak continuity constraints will control the application of the strength and shape of the smoothness. The DA-MRF model for image analysis has proven to be a valuable alternative to the classical MRF model. We follow [2] in the definition of the DA-MRF model, and optimize the model for use in SAR image analysis.

In [3], the prior distribution of the region labels is modeled as $p\left(L_{s} / L_{r}, r \in N_{s}\right)=\frac{1}{Z_{2}} \exp \left[-M U_{2}\left(L_{s} / L_{r}, r \in N_{s}\right)\right]$, where the Gibbs energy function $U_{2}$ is

$U_{2}\left(L_{s} / L_{r}, r \in N_{s}\right)=-\frac{\beta}{M} \sum_{r} \delta\left(L_{s}-L_{r}\right)$.

$\beta$ is a clustering parameter equal to $1.4, Z_{2}$ is a normalizing constant independent of $L_{s}$, and $\delta($.$) is the Kronecker delta. U_{2}$ returns a low value (good) if all the pixels in the neighborhood $N_{s}$ have the same label, and high (not so good) if otherwise.

As is clear from (3), the region labeling is modeled as an isotropic MRF process with local dependencies. In [2] it is argued that in many real-world problems this approximation may not be sufficiently accurate, and this holds true especially in the application of land-cover mapping, where often a great level of detail is needed.

In [2] solutions to this problem have been reviewed. Li focuses solely on the smoothness priors, and his analysis of the different solutions proposed in the literature, such as weak string and membrane [5,6], line process [7], resulted in the so-called discontinuity adaptive smoothness model, of which the before-mentioned models are special instances.

In what follows, we first explain in detail how $\mathrm{Li}$ comes to his DA-MRF prior, after which the integration between the DA-MRF prior and the Gammaobservation model shall be outlined. In its general 1-D form, the smoothness term $U(f)$ is defined as 
$U(f)=\sum_{n=1}^{N} U_{n}(f)=\sum_{n=1}^{N} \lambda_{n} \int_{a}^{b} g\left(f^{(n)}(x)\right) d x$,

being $f$ the signal to be restored, $U_{n}(f)$ the $n$th order regularizer, $N$ is the highest order to be considered and $\lambda \geq 0$ is a weighting factor. A potential function $g\left(f^{(n)}(x)\right)$ is the penalty against the irregularity in $f^{(n-1)}(x)$ and corresponds to prior clique potentials in MRF models. Limiting the model to be of the first order, and considering the general string model, from (4) we can derive

$$
E(f)=U(f / d)=\int_{a}^{b} u(f / d) d x,
$$

where, using for the time being the Gaussian observation model

$$
\begin{aligned}
& U(d / f)=\int_{a}^{b} \chi(x)[f(x)-d(x)]^{2} d x, \\
& u(f / d)=\chi(x)[f(x)-d(x)]^{2}+\lambda g\left(f^{1}(x)\right), \\
& \chi(x) \text { being an appropriate weight function. }
\end{aligned}
$$

The solutions minimizing $U(f / d)$ must satisfy the associated EulerLagrange differential equation $u\left(f, f^{1}\right)-\frac{d}{d x} u^{1}\left(f, f^{1}\right)=0$,

with the boundary conditions $f(a)=f_{a}$ and $f(b)=f_{b}$ which are prescribed constants. Writing out the Euler equation for the Gaussian, one dimensional model yields

$$
2 \chi(x)[f(x)-d(x)]-\lambda \frac{d}{d x} g^{1}\left(f^{1}(x)\right)=0 \text {. }
$$

A potential function $g$ is chosen to be even $(g(\eta)=g(|\eta|))$ and the derivative of $g$ can be expressed as $g^{1}(\eta)=2 \eta h(\eta)$, where $h$ is called an interaction function. The interaction $h(\eta)$ must be small for large $|\eta|$ and approaches to 0 as $|\eta|$ goes to $\infty$. In [2] different Adaptive Interaction Functions (AIF) are proposed, that all satisfy Definition 1:

Definition 1 ([2]): An adaptive interaction function (AIF) $h_{\gamma}$ parameterized by $\gamma$ $(>0)$ is a function that satisfies: (i) $h_{\gamma} \in C^{1}$ (ii) $h_{\gamma}(\eta)=h_{\gamma}(-\eta)$ (iii) $h_{\gamma}(\eta)>0$ (iv) $h_{\gamma}^{1}(\eta)<0 \quad(\forall \eta>0)$ (v) $\lim _{\eta \rightarrow \infty}\left|\eta h_{\gamma}(\eta)\right|=C<\infty$. In section 5 more details on the AIF are given.

Extending (7) to 2-D yields 


$$
\chi[f-d]-\lambda \frac{\partial}{\partial x}\left[f_{x} h\left(f_{x}\right)\right]-\lambda \frac{\partial}{\partial y}\left[f_{y} h\left(f_{y}\right)\right]=0 .
$$

The Euler equation can be solved by minimizing the corresponding energy (5). Using the first order backward difference as an approximation of the first derivative, (5) can be approximated by

$$
E(f)=\sum \chi_{i}\left[f_{i}-d_{i}\right]+\lambda \sum_{i=1}^{m} \sum_{r \in N_{i}} g\left(f_{i}-f_{r}\right),
$$

where $N_{i}$ consists of the neighbors of $i$. From the above, using the gradient-decent method, we obtain the following updating equation:

$$
f_{i}^{(t+1)} \leftarrow f_{i}^{(t)}-2 \mu\left\{\chi_{i}\left[f_{i}^{(t)}-d_{i}\right]-\lambda \sum_{r \in N_{i}}\left(f_{r}^{(t)}-f_{i}^{(t)}\right) h\left(f_{r}^{(t)}-f_{i}^{(t)}\right)\right\},
$$

where $\mu$ is the update strength, and is chosen constant during the optimization process. At each iteration, the label of each pixel is updated based on the contextual information in the label image at the previous itaration. The updating process, which is a deterministic relaxation, starts with a certain initial label configuration $f^{(t=0)}$. A suited stop criteria should be defined.

\section{DA-MRF model with gamma observation statistics}

Since the approach of Rignot and Chellappa deals with classification rather than with segmentation, the values of $L_{s}$ and $f(x)$ in (2) and (7), respectively, are class indexes and have a semantic value rather than a quantitative one. To solve this problem, and to enable one to convert the non-linear equation into a linear one, it is proposed to substitute the class indexes with the mean class-intensity values:

\rangle $d\left\langle_{f_{i}}=f_{i}\right.$.

Using (8), and substituting the Gaussian data distribution with the Gamma distribution

$U_{1}\left(d_{i, j} / f_{i, j}\right)=\frac{N d_{i, j}}{f_{i, j}}-(N-1) \log \left(d_{i, j}\right)+N \log \left(f_{i, j}\right)$

allows us to re-write (7) into

$$
u(f / d)=\frac{N d_{i, j}}{f_{i, j}}-(N-1) \log \left(d_{i, j}\right)+N \log \left(f_{i, j}\right)+\lambda g\left(f_{i, j}^{\prime}\right) .
$$

Writing out the Euler equation for the Gamma case yields

$$
-\frac{N d_{s}}{\left(f_{x, y}\right)^{2}}+\frac{N}{f_{x, y}}-\lambda \frac{\partial}{\partial x}\left(f_{x}\right) h\left(f_{x}\right)-\lambda \frac{\partial}{\partial y}\left(f_{y}\right) h\left(f_{y}\right)=0 \text {. }
$$


The posterior energy can now be estimated by

$$
E(f)=\sum_{i=1}^{m} U_{1}\left(d_{i} / f_{i}\right)+\lambda \sum_{i=1}^{m} \sum_{r \in N_{i}} g\left(f_{i}-f_{r}\right)
$$

and using the classical, deterministic gradient based minimization method yields the following updating scheme (10):

$$
f_{s}^{(t+1)} \leftarrow f_{s}^{(t)}-\mu\left\{\left[\frac{N\left(f_{s}^{(t)}-d_{s}\right)}{\left(f_{s}^{(t)}\right)^{2}}\right]-\lambda \sum_{r \in N_{s}}\left(f_{r}^{(t)}-f_{s}^{(t)}\right) h\left(f_{r}^{(t)}-f_{s}^{(t)}\right)\right\}
$$

The regularizer takes effect when $\lambda>0$. Its strength depends on the qualitative and quantitative shape of the AIF, basically determined by $h_{\gamma}$. For the experiments that follow, the AIF was chosen to be $h_{\gamma}=(1+|\eta| / \gamma)^{-1}$ in both the $x$ and $y$ directions. The main reason is that it allows bounded but non-zero smoothing at discontinuities, useful properties when analyzing speckle images. Additional advantages are that the resulting energy function is convex and that the algorithm becomes computational efficient.

\section{Experimental results}

\subsection{Parameter settings}

Table I should give the reader a feeling about the influence of the various parameters.

Table I. Overview of parameters, their effects, and their suggested values for a 4 look SAR intensity image. The values correspond to those used to produce the results.

\begin{tabular}{|c|l|l|l|c|}
\hline Parameter & \multicolumn{1}{|c|}{ Function } & \multicolumn{2}{|c|}{ Effect } & $\begin{array}{c}\text { Suggested } \\
\text { value }\end{array}$ \\
\cline { 3 - 5 } & & $\begin{array}{l}\text { within order } \\
\text { of magnitude }\end{array}$ & between 0.0.m. & Filtering \\
\hline$\lambda$ & $\begin{array}{l}\text { Relation between observation and } \\
\text { regularization model }\end{array}$ & Small & $\begin{array}{l}\text { large: strong } \\
\text { smoothing. } \\
\text { Low: none. }\end{array}$ & 0.001 \\
\hline$\gamma$ & Choice of regularization strength. & Strong & Strong & $0.1-3$ \\
\hline$\mu$ & $\begin{array}{l}\text { Update strength (optimization } \\
\text { procedure) }\end{array}$ & None. & $\begin{array}{l}\text { Large: } \\
\text { unstable; } \\
\text { small: slow } \\
\text { convergence }\end{array}$ & 50 \\
\hline$K$ & $\begin{array}{l}\text { Number of iterations } \\
\text { (optimization procedure) }\end{array}$ & & & 100 \\
\hline
\end{tabular}




\subsection{Data description}

In order to assess the usefulness of the proposed method, experiments have been done on portions of a 4-Look SAR intensity image ( $\mathrm{HH}$ polarization) of a Flevoland (NL) scene, acquired during the Maestro I campaign in 1989. The original test image is shown in Fig. 3a.

\subsection{Comments on results}

Experimental results are reported in Fig. 3. Fig. 3b shows that, applying a low $\lambda$ value, the final result tends to convert to the original data. With an increasing $\lambda$, clustering of regions gets stronger (Fig. 3c). The strong effect of $\gamma$, reported in table I, can be seen in Fig. 3c and d.

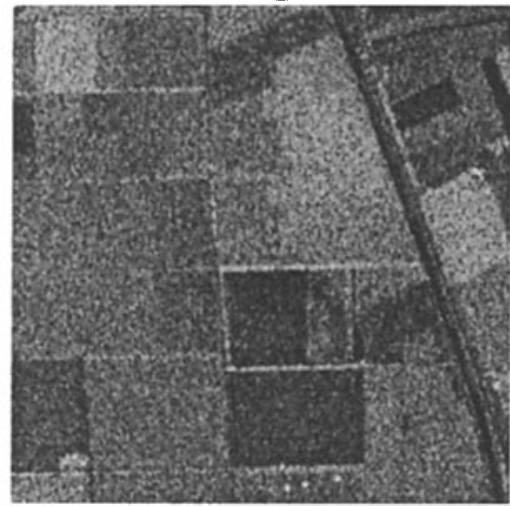

a.

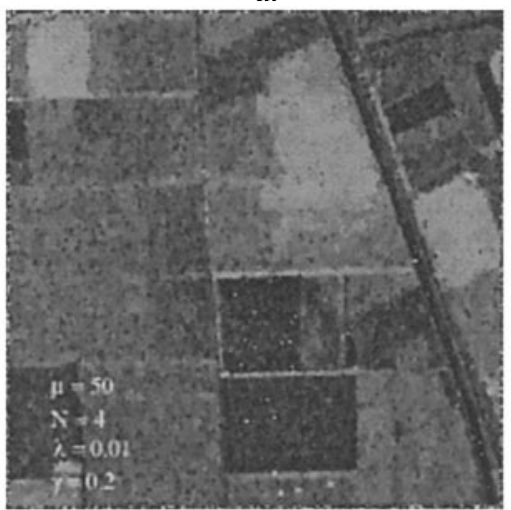

c.

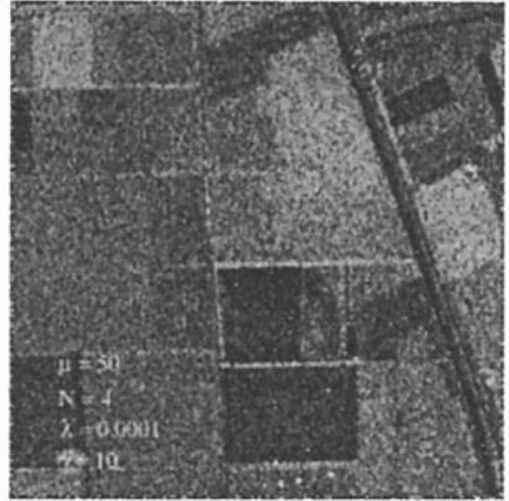

b.

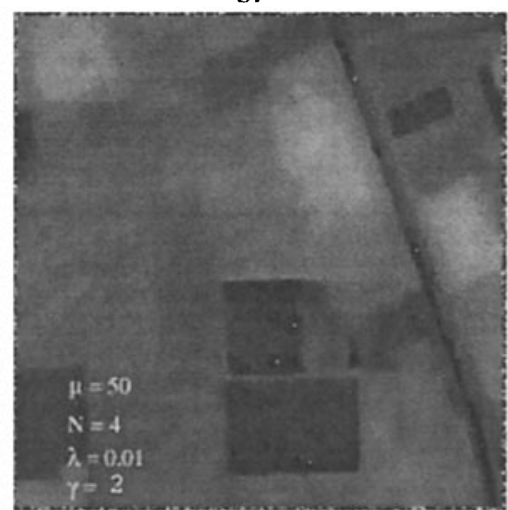

d.

Fig. 3. Results. a) $256 \times 256$ Portion of a Flevoland 4 look SAR intensity image (HH polarization); b) Light filtering: the small $\lambda$ value $(0.0001)$ practically "switches off" the regularizing term; c) And d) show the effect of a small and large $\gamma$ value, respectively. All results in the image were obtained after 100 iterations. 


\section{Discussion and conclusions}

In this paper, an approach has been presented for the analysis of synthetic aperture radar (SAR) images that preserves better fine structures and borders in the image than classical methods. The method uses the discontinuity adaptive MRF model in which the Gaussian observation model has been replaced by a gamma distribution. This resulted in a new algorithm that is more suited to the segmentation of SAR images if one is interested in preserving details. The clustering effect of regions with homogeneous back scatter signals is principally determined by mainly two parameters whose function is easy and intuitively to understand.

Results on various real-world data sets have shown that the method proposed in this paper is a useful tool for the analysis and interpretation of SAR images. However, it is stressed that at the end of the day it remains the end-user to decide what level of detail is required.

The software of the proposed algorithm for Windows 95 will be made available via URL http://dibe.unige.it/TMR_Smits. Readers are encouraged to try the method on their own imagery and to report their findings.

\section{Acknowledgements}

The authors with to thank Dr. A. Freeman of NASA/JPL for providing the SAR data. This work was supported by the European Community program Training and Mobility for Researchers (Marie Curie Fellowship) under contract ERBF MBICT 95257.

\section{References}

[1] Li, S.Z. (1995b), Markov Random Field modeling in computer vision, Springer Verlag, New York.

[2] Li, S.Z. (1995), Discontinuity-adaptive MRF prior and robust statistics: a comparative study, IEEE Trans. on Pattern Analysis and Machine Intelligence 17(6), 576-586.

[3] Rignot, E., Chellappa, R. (1993). Maximum a posteriori classification of multifrequency, multilook, synthetic aperture radar intensity data, J. Opt. Soc. Am. A, Vol. 10, No. 4, pp. 573-582.

[4] Smits P.C. and S. Dellepiane (1996), "Information fusion in a Markov Random Field based image segmentation approach using adaptive neighbourhoods," 13th Int. Conf. on Pattern Recognition, Vienna, August 1996, pp. 570-575.

[5] Blake (1983), The least disturbance principle and weak constraints, Pattern Recognition Letters, Vol. 1, pp. 393-399.

[6] Blake A. and A. Zisserman (1987), Visual reconstruction. Cambridge, MA: MIT Press.

[7] Geman S. and Geman D. (1984), Stochastic relaxation, Gibbs distributions and the Bayesian restoration of images, IEEE Trans. Pattern Anal. Machine Intell., Vol. PAMI-6, nov. 1984, pp. 721-741. 